\title{
Design optimization for energy-efficient pulse-switching networks in carrier-injection based Si-photonics
}

\author{
Clemens J. Krückel ${ }^{1,2 *}$, Hanna Becker ${ }^{3}$,Martijn J.R. Heck, \\ Joris Van Campenhout ${ }^{2}$, and Dries van Thourhout ${ }^{1,2}$ \\ ${ }^{1}$ Photonics Research Group, INTEC, Ghent University-IMEC, Ghent B-9000, Belgium \\ ${ }^{2}$ IMEC, Kapeldreef 75, Leuven B-3001, Belgium \\ ${ }^{3}$ Department of Engineering, Aarhus University, Denmark \\ *E-mail: clemens.kruckel@ugent.be
}

Keywords: integrated optics devices, optical switching devices, optical networks-on-chip, silicon photonics

\begin{abstract}
We compare pulse-switching operations in MZI- and ring-switches both experimentally and based on large-signal circuit simulations. With a modification in switch design and with optimization of phase modulator lengths, we show high-speed switches with potential for an over $3 \mathrm{~dB}$ improvement in energy consumption.
\end{abstract}

\section{Introduction}

All-optical writing of spintronic memory elements has great potential for high-speed data storage [1]. The spintronic memory state is changed once the delivered optical energy exceeds a critical threshold value. In the EU-project SPICE [2], we work towards an optical routing network that allows addressing of these spintronic elements individually. The requirements for the reconfigurable networks are the following: first, the network needs to provide an optical pulse with high optical fluence $\left(5.8 \mathrm{~mJ} / \mathrm{cm}^{2}\right)$ at the output port of the network. This requires, besides focusing grating couplers at the output [3] and low circuit losses, the potential to route ps pulses. Secondly, we want to enable energy-efficient data storage. Thus, the energy consumption per switching operation has to be minimized. Thirdly, the network should support 100-MHz switching speed.

The fabrication of these reconfigurable networks within the silicon photonics platform offers foremost the advantage of a well-established fabrication process. In addition, utilizing carrier-injection technology, allows for the fabrication of efficient switches with low insertion loss [4] that support sufficient switching speed for our application. A drawback of this technology, though, is the constant static power consumption when active. Switching short pulses however, negates this drawback as the static power consumption becomes insignificant, due to the short gating time. For this reason, we investigate if we can find a sweet spot for carrier-injection technology.

Here, we demonstrate the importance of switch architecture, design and driving scheme. We compare pulse switching by Mach-Zehnder interferometer (MZI) switches and ringswitches and suggest how to further improve performance towards energy-efficient optical pulse switching above 100$\mathrm{MHz}$ speed.

\section{Pulse switching}

Photonic switches designed in a MZI architecture offer an optical bandwidth beyond $100 \mathrm{~nm}$ [4]. This optical bandwidth is sufficient to switch fs-pulses. For switching of spintronic memory elements these shorter pulses are generally favorable [1]. Pulse switching is demonstrated with a $1 \times 2$ MZI-switch using $500 \mu \mathrm{m}$ long phase shifters. In the experiment, as illustrated in Fig. 1, the light pulses of $\sim 40 \mathrm{fs}$ at a repetition rate of $20 \mathrm{MHz}$ are switched between the two output ports (1 and 2), such that every second pulse is routed to output port 2 . The electrical driving signal utilizes a pre-emphasis-scheme which leads to significant improvement in switching speed [4]. Moving from a square waveform to a $2 \mathrm{~V}$ pre-emphasis driving-scheme results in a more than 50 times faster activation (from $20 \mathrm{~ns}$ to $0.4 \mathrm{~ns}$ ). For the demonstration of ps-pulse switching in ring-switches, we use an add-drop ring system [6]. The ring is designed with a 15 - $\mu \mathrm{m}$ radius and doping of $\sim 56 \%$ of the rings circumference provides an effective pin-diode length of $\sim 53 \mu \mathrm{m}$. It should be noted, that the pin-diode design is the same for both switch architectures. The transmission scans of thru- and drop-port,

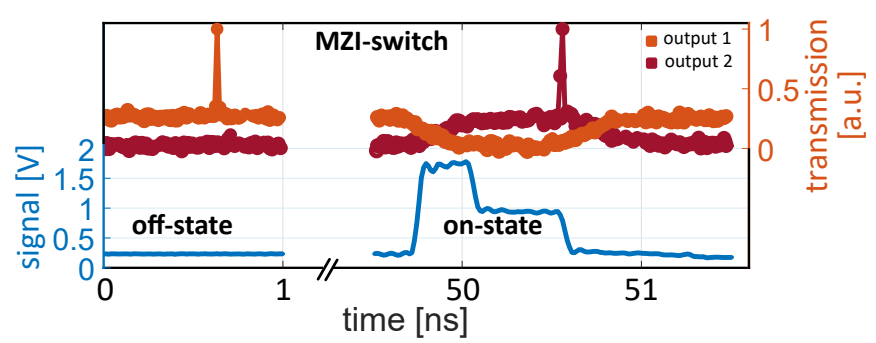

Fig. 1 Pulse switching in a $1 \times 2$ MZI-switch with $500 \mu \mathrm{m}$ phase-shifter length. Switch activation with a pre-emphasis driving signal. Beside the pulses, CW-light was switched in order to illustrate the transient response during switching operation. 


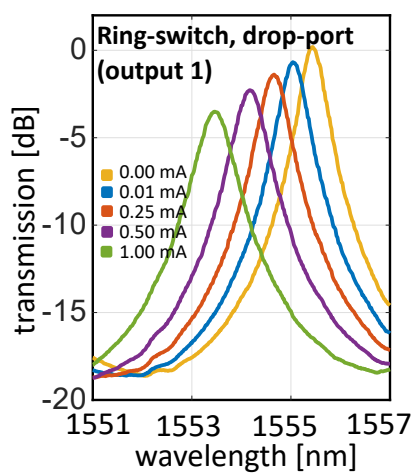

a)

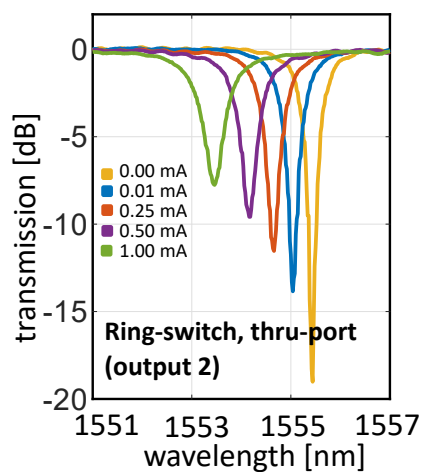

b)
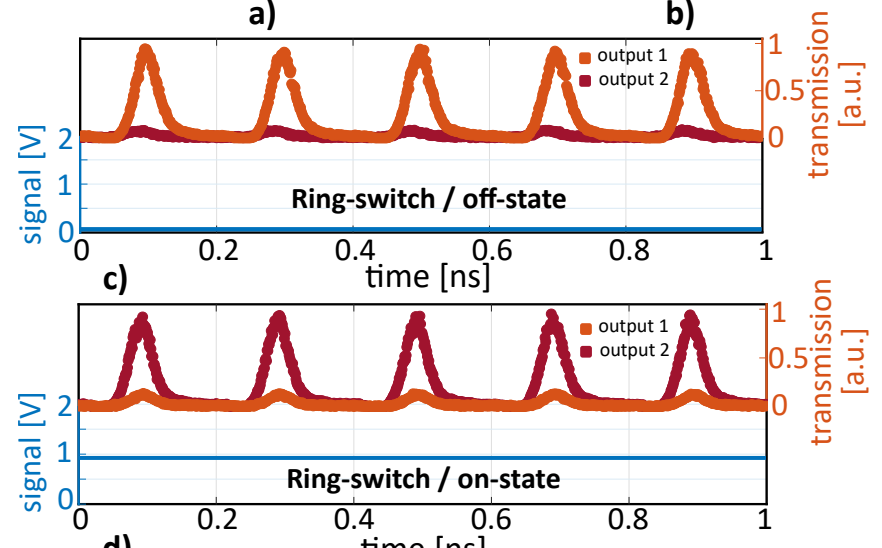

d)

Fig. 2 a) and b) Transmission spectrum of drop-port and through-port of a ring-switch $(15 \mu \mathrm{m}$ radius, $\sim 53 \mu \mathrm{m}$ diode length) at various DC diode currents. c) Transmission of the ring-switch at both output ports in off-state. d) Transmission of the ring-switch at both output ports when operated in the on-state $(0.25 \mathrm{~mA}$ diode current).

referred to as output 1 and 2, are shown in Fig. 2.a+b at various injected diode currents. The ring exhibits a lower optical bandwidth compared to the MZI, yet it is sufficient to switch pulses longer than 5 ps. Even though a lower Q-factor would increase the optical bandwidth, a larger wavelength shift would be required for switching, making the device less energy-efficient. For the pulse switching, the centre-wavelength of the pulsed spectrum is tuned into off-state resonance. In Fig. 2.c the switch is operated in the off-state and pulses are present at output 1. In on-state, pulses are routed to output 2, as shown in Fig. 2.d.

\section{Insertion loss and extinction ratio}

To compare the insertion loss and extinction ratio of MZIand ring-switches, we look at the transmission of the switch at both output ports versus injected diode current. Fig. 3.a and 3.b show the transmission curve for the ring and a 500 $\mu \mathrm{m}$ MZI-switch respectively. The transmission values of the ring-switch are extracted from a pulse switching experiment and referenced to the $\mathrm{CW}$ insertion-loss off-resonance. We see comparable insertion loss in off-state at $0 \mathrm{~mA}$ diode current with a similar extinction ratio of around $10 \mathrm{~dB}$. The switch performance is however different in the on-state when maximum power is available at output 2 . While the on-state in the

MZI-switch is achieved at a fixed current value of $\sim 0.5 \mathrm{~mA}$ (inducing a $\pi$-phase shift), the ring-switch is fully activated above $0.23 \mathrm{~mA}$. We define activation of the ring-switch as the minimum required current to reach an extinction ratio above $8 \mathrm{~dB}$ which is sufficient for the chosen application, yet maximizing throughput. Additionally, it can be observed that the ring shows a lower insertion loss in the on-state. For the MZIswitch this insertion loss originates from the carrier-absorption in the phase shifter. This is not the case for the ring resonator when operated as mentioned above (switching off-resonance) but relevant when switching in resonance. As the insertion loss is an important parameter of the switch, we investigate how the length of the phase shifter can be changed to maximize the throughput of the system.

For that we studied stand-alone phase shifters and measured the absorption loss for eight different lengths from 250 to 1400 $\mu \mathrm{m}$ as a function of diode current. In Fig. 3.c the absorption is plotted versus diode length for off- and on-state. The onstate is defined for current values that induce a $\pi$-phase shift. In on-state we see a reduction in loss with length which originates from the smaller carrier-density in longer diodes. We see a good match to the theory [6]. The deviation might arise due
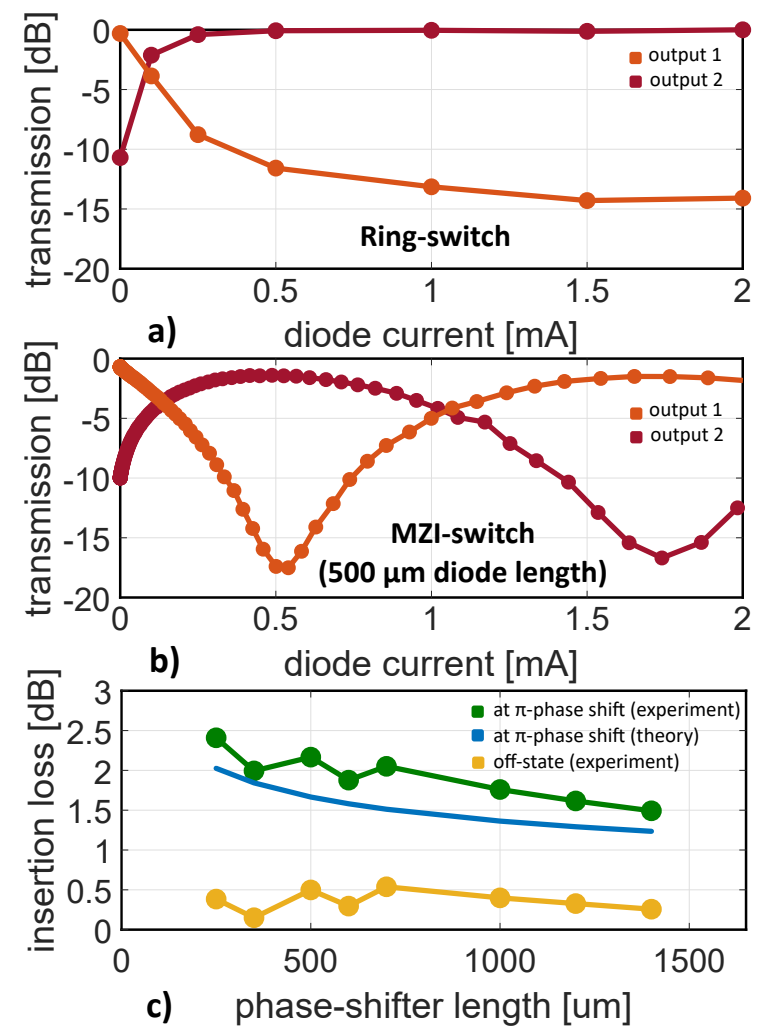

Fig. 3 a) Transmission at both output ports of the ring-switch (15 $\mu \mathrm{m}$ radius, $\sim 53 \mu \mathrm{m}$ diode length) as a function of applied diode currents. b) Transmission at both output ports of the MZI-switch (500 $\mu \mathrm{m}$ phase shifter) as a function of applied diode currents. c) Insertion loss of stand-alone phase-shifters in off-state operation ( $0 \mathrm{~mA})$ and when inducing a $\pi$-phase shift. The theoretical absorption loss at $\pi$-phase shift is also shown. 
to measurement uncertainties and is visible in both on- and offstate. In summary, for a lower insertion loss longer diodes are advantageous, though sacrificing footprint.

\section{Energy consumption and switching speed}

In order to study the energy consumption in phase shifters, we determined the required diode current and voltage of a phase shifter to reach a $\pi$-phase shift. Figure 4 .a shows these values as a function of phase shifter length allowing us to compute the diodes static power consumption in steady-state operation. The required current is important to be reduced, as it impacts the dynamic energy consumption. We see that the current value is ranging from 1.4 to $0.2 \mathrm{~mA}$ with increased diode length making longer diodes more energy efficient. This effect can be explained by the nonlinear relation between carrier-density and optical phase shift. The deviation of the on-state current of the $500 \mu \mathrm{m}$ phase shifter shown in Fig. 2.b from the expected value is explained by fabrication tolerances yielding an unbalanced MZI. In comparison to the stand-alone phase shifters, the ring switch needs only $0.23 \mathrm{~mA}$ for activation.

We evaluate the dynamic energy consumption with a largesignal model [8]. The used equivalent circuit model for each

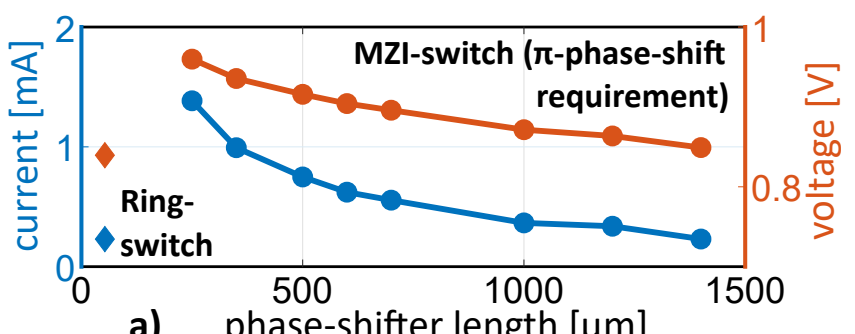

a) phase-shifter length $[\mu \mathrm{m}]$
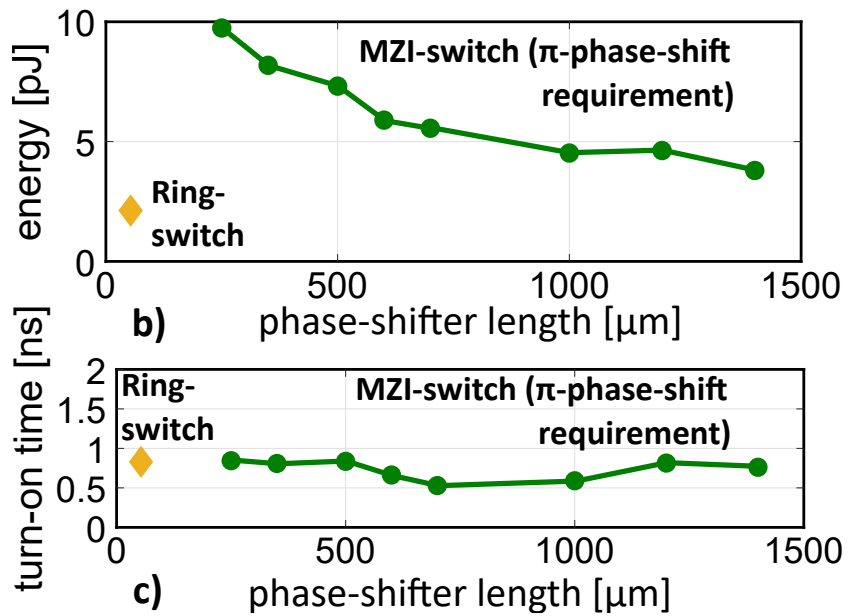

Fig. 4 a) Evaluated driving current and voltage to achieve switching in the Ring-switch and in MZI-switches. Requirement for MZI-based switching is a $\pi$-phase shift in the phaseshifter. b) Energy consumption per switching operation as a function of phase shifter length. The pre-emphasis driving signal was optimized for the lowest energy consumption (preemphasis voltage below $2 \mathrm{~V}$ for all phase-shifters). c) Diode length dependent turn-on time at pre-emphasis driving signal that yields minimum energy consumption. diode length is based on measurement data for series resistance, ideal diode and capacitance and explained in more detail in [9]. The simulations determine the energy required to inject the needed charge into the phase shifter. We see a strong relation of consumed energy and driving-scheme. In essence, pre-emphasis activation leads to energy savings of more than $40 \%$ owing to the reduction of charge dissipation at higher driving currents. As Joule heating increases with pre-emphasis voltage, there is a driving voltage that yields minimum activation energy. In Fig. 4.b we summarize this minimum switching energy, defined as the required energy to reach on-state, versus diode length. It is important to highlight that this minimum dynamic energy is exclusively limited by the charging energy of the diode capacitance [9]. The lower current-target for a $\pi$ phase shift (Fig. 4.a) seems to promote the energy efficiency of longer diodes. The clear trend indicates a reduction of energy consumption towards longer diodes by a factor of two. The ring-switch has a lower current-target for activation. Together with the lower capacitance of the short ring-diode, this results in $\sim 50 \%$ reduced energy consumption compared to the longest phase-shifter.

Another impact of the larger driving current with increased preemphasis voltage is the increase in switching speed. From our large-signal simulation we extracted the turn-on time at the preemphasis voltage leading to minimum energy consumption. All diodes have short sub-ns turn-on time around $0.7 \mathrm{~ns}$ as shown in Fig. 4.c.

\section{Conclusion}

We showed that the diode length is an important design parameter in optimizing carrier-injection based switches. The nonlinear relation between diode length and required carrier-density for a $\pi$-phase shift leads to a $\sim 50 \%$ reduction in energy consumption for longer diodes. Although footprint is sacrificed, longer diodes show additionally an improvement in absorption loss ( $\sim 1.5 \mathrm{~dB}$ in on-state). Using a ring switch will combine small footprint with switching energies below $3 \mathrm{pJ}$. The ring design yields a $\sim 50 \%$ more energy efficient switch, compared to the MZI structure. However, the switchable pulse width is limited by the low optical bandwidth of the ring, but it is still adequate for pulse-switching to address MTJs. In general, using pre-emphasis voltages significantly reduces energy consumption and allows sub-ns switching time for all systems. We conclude that considering these design optimizations, carrier-injection based silicon-photonics is a suitable platform for energy-efficient pulse switching with low insertion loss.

\section{Acknowledgments}

This project has received funding from the European Union's Horizon 2020 research and innovation programme under grant agreement No 713481 . 


\section{References}

[1] J.-Y. Chen et al., 'All-Optical Switching of Magnetic Tunnel Junctions with Single Subpicosecond Laser Pulses', Phys. Rev. Appl., 2017, 7, (2), pp. 021001-6

[2] http://spice-fetopen.eu/

[3] H. Becker et al., 'Out-of-plane Focussing Polarization Control Grating Couplers for Photonic-Spintronic Integration', Conference on Lasers and Electro-Optics (CLEO) Europe, Munich, Germany, June 2019

[4] J. Van Campenhout et al., 'Low-power, $2 \times 2$ silicon electro-optic switch with 110-nm bandwidth for broadband reconfigurable optical networks', Op. Exp., 2009, 17, (26), pp. 24020-24029

[5] Q. Xu et al., '12.5 Gbit/s carrier-injection-based silicon micro-ring silicon modulators', Op. Exp., 2007, 15, (2), pp. $430-436$

[6] W. Bogaerts et al., 'Silicon microring resonators', Laser Photonics Rev., 2012, 6, (1), pp. 47-73

[7] R. Soref et al., 'Electrooptical Effects in Silicon', IEEE J. Quantum Electron., 1987, 23, (6), pp. 123-129

[8] R.F. Pierret et al., 'Semiconductor Device Fundamentals', Addison Wesley, 1996

[9] C.J. Krückel et al., 'Towards High-Speed EnergyEfficient Pulse-Switching Networks Implemented in Carrier-Injection-Based Si-Photonics', Conference on Lasers and Electro-Optics (CLEO) Europe, Munich, Germany, June 2019 